\title{
Design of Off-Midplane Launcher (LH3) Based on Velocity Space Synergy for Alcator C-Mod ${ }^{*}$
}

\author{
Syun'ichi SHIRAIWA, Seung Gyou BAEK, Paul T. BONOLI, Ian FAUST, Julien HILLAIRET ${ }^{1)}$, \\ Orso MENEGHINI ${ }^{2)}$, Ronald R. PARKER, Gregory M. WALLACE, James R. WILSON ${ }^{3)}$, \\ Robert W. HARVEY ${ }^{4)}$ and Alexander P. SMIRNOV ${ }^{5}$ \\ Plasma Science Fusion Center, MIT, MA, 02139, USA \\ ${ }^{1)}$ CEA, IRFM, F-13108 Saint Paul-lez-Durance, France \\ ${ }^{2)}$ Oak Ridge Associated Universities, Oak Ridge, Tennessee, USA \\ 3) Princeton Plasma Physics Laboratory, Princeton, New Jersey 08543, USA \\ ${ }^{4)}$ CompX, Del Mar, California 92014, USA \\ ${ }^{5)}$ M.V. Lomonosov Moscow State University, Moscow, Russia
}

(Received 10 December 2012 / Accepted 13 April 2013)

\begin{abstract}
A new LH launcher (LH3) is designed for Alcator C-Mod to increase the net LHCD power to 2MW. With the existing launcher (LH2), LH3 aims to enhance the single pass power absorption to improve LHCD efficiency at high density. For this purpose, launcher design parameters are surveyed to maximize the synergistic effect between LH2. Ray-tracing is extensively used and the launcher location and the launched $N_{\|}=c / v_{\|}$are optimized. At the line averaged density of $1.4 \times 10^{20} \mathrm{~m}^{-3}$, it is predicted that the combination of two launchers can reduce the parasitic edge loss dramatically, and therefore can increase the LH driven current by about 50 - $60 \%$ compared to cases in which two launchers are used separately. Based on the parameter survey, an off-midplane launcher (LH3) is designed to be located $15 \mathrm{~cm}$ above mid-plane. LH3 employs 8-way power splitters to improve the antenna-plasma coupling. The antenna-plasma coupling simulation is performed using COMSOL and ALOHA codes, predicting $90 \%$ of RF power coupling when the launcher front density is high enough ( $\geqslant n_{\text {cutoff }}$ ).
\end{abstract}

(c) 2013 The Japan Society of Plasma Science and Nuclear Fusion Research

Keywords: tokamak, RF modeling, lower hybrid current drive

DOI: $10.1585 /$ pfr.8.2402143

\section{Introduction}

In lower hybrid (LH) current drive, the LH wave is absorbed via electron Landau damping (ELD) and electrons are accelerated to the parallel direction with respect to the magnetic field [1], which makes it an ideal tool for far off-axis current drive for tokamak experiments including ITER [2]. LHCD has a high current drive efficiency and has been a main current drive tool for sustaining tokamak plasmas in steady-state. On Alcator C-Mod, fully non-inductive discharges have been already obtained with $n_{\mathrm{e}}=0.5 \times 10^{20} \mathrm{~m}^{-3}$, and $B_{\mathrm{T}}=5.4 \mathrm{~T}$ [3], which are close to what is expected on ITER steady state operation. At this density, we have demonstrated to sustain plasmas for several current relaxation time with flat or weakly reversed q profiles and to develop ITB in the electron temperature profile, providing an opportunity to study transport and stability at ITER-relevant steady-state regimes as well as LH wave physics and current profile control. The engineering LH current drive efficiency is $2-2.5 \times 10^{19} \mathrm{~A} / \mathrm{Wm}^{2}$, which is consistent with what is predicted on ITER.

However, as the density increases, the LHCD effi-

author'se-mail: shiraiwa@psfc.mit.edu

$\left.{ }^{*}\right)$ This article is based on the presentation at the $22 \mathrm{nd}$ International Toki Conference (ITC22). ciency was found to decrease more quickly than predicted by a ray-tracing code in a diverted configuration $[4,5]$. Better understanding and mitigating this unexpected result has been a major topics for recent LHCD experiments on Alcator C-Mod and worldwide LHCD research activities [6]. On C-Mod, we developed two independent computational models, one is based on a ray-tracing code expanded to include SOL plasmas $[4,7,8]$, and the other is a finite element method (FEM) based full wave code $[9,10]$, which can also treat complicated SOL plasma geometry directly. Both of these models have better treatment of parasitic edge losses. Also experimentally, detailed survey of LH wave spectra has been performed at various location around tokamak plasmas [11].

A picture emerged from these studies is that as the density increases, more and more edge parasitic mechanisms start to contribute significantly to power loss of LHCD due to a weak single pass power absorption, diminishing the power absorbed via ELD. In short, the result of these studies can be summarized as follows. Figure 1 shows experimentally observed hard X-ray (HXR) emission from LH fast electrons as the function of density, with predictions of the ray-tracing and fullwave codes. 
Hard X-ray from LH fast electrons

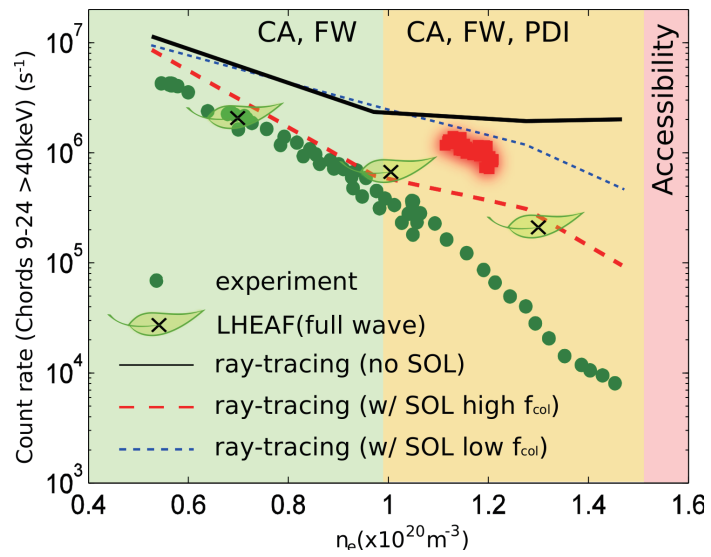

Fig. 1 Experimental observation of LH generated hard Xray emission and predictions of simulation codes. (Green) experimental data, (black) GENRAY/CQL3D raytracing/Fokker-Planck code [7, 8], (red/blud) GENRAY/CQL3D with SOL plasmas, and (leaf symbol) LHEAF full wave code [12]. Red symbols are experimental HXR emission measured on a discharge without a strong PDI activity.

The experimental data shows an exponential decrease of HXR emission in the entire range of density (0.5-1.5 $\times 10^{20} \mathrm{~m}^{-3}$ ), and the prediction from a simple ray-tracing without SOL plasmas (black curve) has a large discrepancy, which becomes wider as the density increases. With the improved codes, the agreement gets better, but still there is a discrepancy remains at density above $1.0 \times$ $10^{20} \mathrm{~m}^{-3}$. In this density regime (denoted by orange area), the LH spectrum survey revealed that edge parametric decay instabilities [13] can be excited, suggesting this nonlinear effect is a possible cause of the remaining discrepancy [11]. Physics processes used in codes, collisional damping and full wave spreading of spectrum, become more prominent when the single pass absorption is weak. Also, there is no clear evidence that PDI occurs in front of launcher, inducing a prompt loss of launched power, on C-Mod. We also observed an increase of HXR emission when PDI level was small even at $n_{\mathrm{e} 0}>1 \times 10^{20} \mathrm{~m}^{-3}$ (red symbols). Although the condition to reproduce this improvement is not yet clear, it suggests a close connection between the suppression of PDI and the improvement of LHCD at high density.

This view has a significant impact both for future LH experiment on Alcator C-Mod and LHCD on ITER. For Alcator C-Mod, accessing advanced tokamak (AT) regimes with a large bootstrap current fraction $\left(f_{\mathrm{BS}} \sim\right.$ $50 \%$ ) requires to operate a tokamak at a density above $1 \times 10^{20} \mathrm{~m}^{-3}$ with $T_{\mathrm{e} 0} \sim 5 \mathrm{keV}$ [14]. Although target plasmas with these parameters have been produced in C-Mod both by mode-converted ICRF heating as well as by operating in I-Mode [15], it would be difficult to sustain such a plasma without recovering current driven efficiency of LHCD. For ITER, if the degradation is caused by the low single pass power absorption, the current drive efficiency may recover naturally, since ITER has a strong single pass absorption. However, if it is caused by a prompt loss of LH wave in front of launcher as conjectured in Ref. [16], it might be still an issue.

Motivated by this background, during the design phase of the additional launcher (LH3) to increase the total LH power to over $2 \mathrm{MW}$ level, we explored possibilities to dramatically enhance the single pass absorption. In this paper, we describes the method we employed to realize high single pass absorption on C-Mod and the RF design of LH3 coupler.

\section{Velocity Space Synergy}

The reason why the $\mathrm{LH}$ waves experience multiple reflections before its power is absorbed by ELD is that the wave parallel phase velocity, $v_{\|}$, needs to slow down enough so that the wave can interact with bulk electrons and extract non-thermal tail electrons. If the shape of distribution function at low velocity (around $3 \times v_{\text {th }}$, where $v_{\text {th }}$ is the electron thermal velocity) can be modified by some other methods, the LH wave can be absorbed more quickly. This concept is termed velocity space synergy, and several schemes to generate such a synergy have been developed including combining of EC and LH waves [17], and using LH waves with different frequencies simultaneously [18].

The scheme employed for LH3 is to use an offmidplane launcher. This approach was tested on PLT and JT-60U [19], with a positive result, and the experiment on C-Mod will be the first attempt to use it to solve the issue at high density. To illustrate how this approach works, we consider the LH wave launched by a single off-midplane launcher for a moment.

The wave launched with a finite poloidal angle is known [20] to experience very different change of the parallel wave number, $N_{\|}=c / v_{\|}$compared to a ray launched from the mid-plane. If the sign of poloidal angle is properly selected, $N_{\|}$will up-shift quickly, resulting in strong damping. For a nominal direction of toroidal field and plasma current for Alcator C-Mod, this occurs when a launcher is located above the mid-plane. Figure 2 illustrates the launch poloidal angle dependence of $\mathrm{LH}$ wave propagation in the phase space. The wave absorbed from mid-plane $(0 \mathrm{deg})$ does not experience a large slow down of the phase velocity as it propagates through the plasma and comes out to SOL plasma. On the other hand, the wave launched at high poloidal angle (60 deg. and $90 \mathrm{deg}$.) slows down quickly (high $N_{\|}$upshift), and during the first pass, the trajectory reaches the critical velocity, $3 v_{\text {th }}$, at which the absorption by ELD takes place.

The wave power which is not absorbed during the first pass can be absorbed via edge parasitic absorption, which does not contribute to the current generation. In order to suppress the parasitic absorption, it is preferable to launch the wave with a high poloidal angle. However, since the 


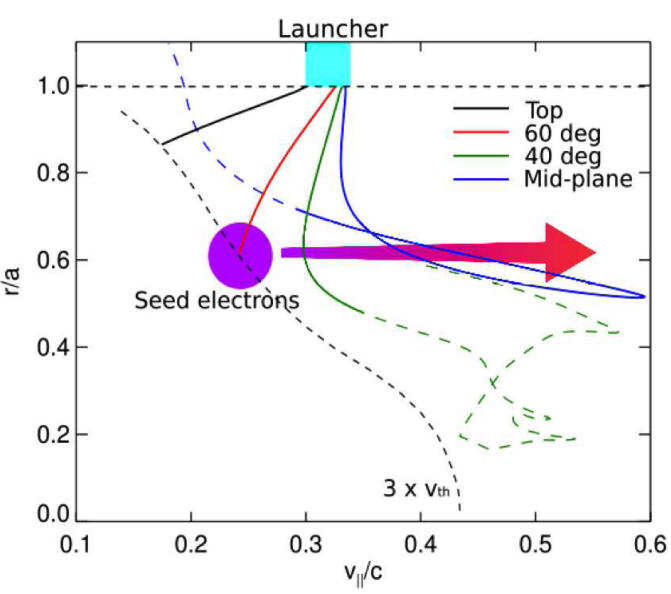

Fig. 2 Phase space trajectory of LH rays launched from four different poloidal angles. Black, red, green, and blue curves shows the wave launched with the poloidal angle of $90 \mathrm{deg}, 60 \mathrm{deg}, 40 \mathrm{deg}$, and $0 \mathrm{deg}$ measured from the midplane, respectively.

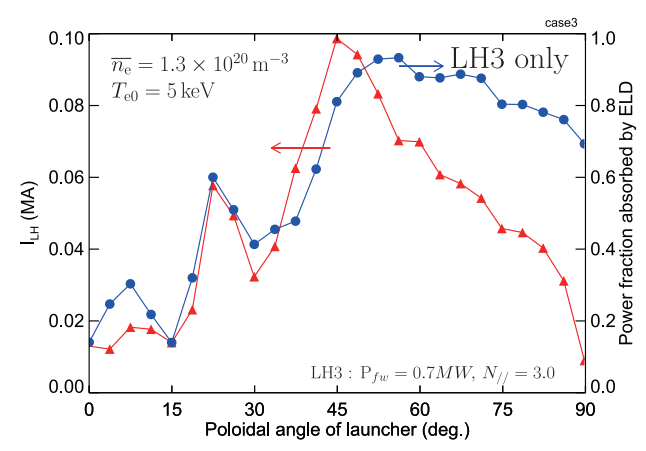

Fig. 3 Predicted driven current and the fraction of LH power absorbed during the first pass, when off-midplane launcher is used along. An I-mode plasma with $n_{\mathrm{e}} 1.3 \times 10^{20} \mathrm{~m}^{-3}$ and $T_{\mathrm{e} 0} \sim 5 \mathrm{keV}$ is used as a target plasma, and LHCD power of $700 \mathrm{~kW}$ at $N_{\|}=3$ is injected.

current drive efficiency of LHCD is inversely proportional to $N_{\|}^{2}$, launching the $\mathrm{LH}$ wave with too high poloidal angle is also not efficient. Figure 3 shows where the optimum efficiency is obtained. In this figure, we evaluate the driven current using the ray-tracing with collisonal edge absorption. An I-mode with $n_{\mathrm{e}} \sim 1.3 \times 10^{20} \mathrm{~m}^{-3}$ and $T_{\mathrm{e} 0} \sim 5 \mathrm{keV}$ is used as a target plasma in this and following simulations. SOL parameters, which determine the strength of parasitic edge absorption, are based on the previous study to reproduce the experimental result. It is shown that too high or too low poloidal angle is not suitable for current generation as expected, and about $45 \mathrm{deg}$ is predicted to have the highest driven current.

\section{Parameter Space Survey to Max- imize the Synergistic Effects with LH2 \\ Although the off-midplane launcher can produce a}

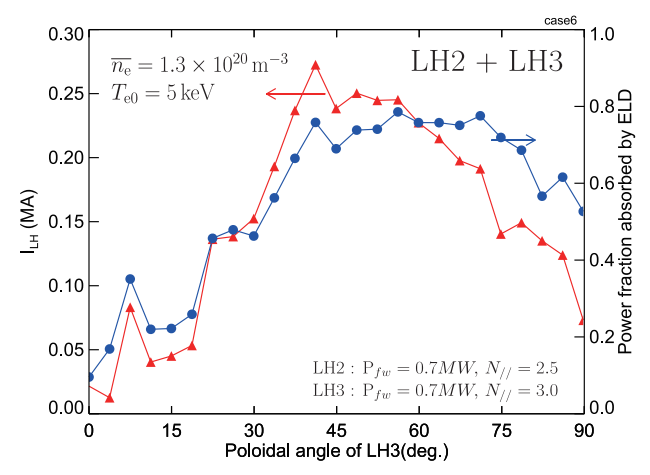

Fig. 4 Predicted driven current and the fraction of LH power absorbed during the first pass, when off-midplane launcher used together with the existing mid-plane launcher. The parameters of the target plasma is the same as Fig. 3.

strong single pass absorption, the current driven by LH wave remains modest due to the high $N_{\|}$up-shift. However, as mentioned earlier, since the wave launched by the off-midplane launcher produces seed electrons, the $\mathrm{LH}$ wave from the mid-midplane can interact with these seed electrons and can be absorbed. Since Alcator C-Mod has already a mid-plane launcher (LH2) installed, we evaluate the driven current when the off-midplane launcher is combined with LH2 (Fig. 4), and scan the position of the new launcher in the same way as shown in Fig. 3 (the power from two launchers are both $700 \mathrm{~kW}$ ). As seen in Fig. 4, although the wave from LH2 has very small single pass power absorption ( 0 deg.), the fraction of $\mathrm{LH}$ power absorbed by ELD reaches $\sim 80 \%$, when the off-midplane launcher is located at high enough poloidal angle. Note that the fraction of power absorbed by ELD is similar between Fig. 4 and Fig. 3, showing that it is mostly determined by the power from off-midplane launcher and the power launched from LH2 is also absorbed more strongly when the poloidal angle of LH3 is high. When LH3 is located at $45 \mathrm{deg}$., the predicted driven current is about $250 \mathrm{kA}, 2.5$ times higher than the case using LH3 alone and 5 times higher than the case using LH2 alone.

\section{RF Coupler for LH3}

Based on the previous survey, the new launcher is designed to be located $15 \mathrm{~cm}$ above mid-plane of Alcator CMod. In this section, we describe a RF coupler (plasma facing component of the launcher) design developed for LH3 and the evaluation of its coupling performance. In LH2 launcher, we employed 4-way splitter as the final stage power splitting module [3]. The 4-way splitter splits the microwave power equally in the poloidal direction, and 16 of the splitter were stacked in the toroidal direction, consisting the $4 \times 16$ active wave guides. The advantage of this design was to realize wide range of $N_{\|}$, while keeping the feeding structure relatively simple. However, since the evenness of power splitting depends on the impedance of 


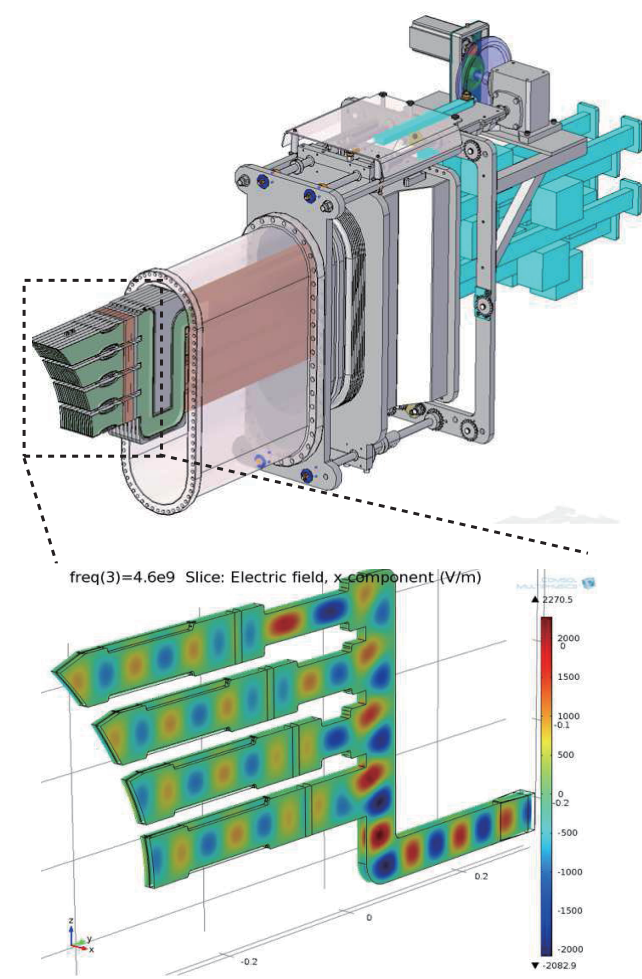

Fig. 5 Schematics LH3 launcher. The launcher is located about $15 \mathrm{~cm}$ above the mid-plane and is equipped with $4 \times 16$ active wave guide. The width of wave guide is selected to $5.5 \mathrm{~cm}$.

plasma load on four rows, the difference of density profiles in front of four rows affect the evenness of power splitting In LH3, we added bi-junctions [24] to each of four output ports of 4-way splitter, making total 8-way power splitting. This modification reduces the power reflection, and thereby the sensitivity of power splitting evenness to the poloidal variation of density profile. It also allows for further simplification of feeding structure.

Figure 5 shows a schematics of LH3 and its 8-way splitter. Eight of 8-way splitters, each of which is powered by $4.6 \mathrm{GHz} 250 \mathrm{~kW}$ klystron via the standard WR187 waveguide, are stacked in the toroidal direction. The output waveguide of 8-way splitter has the dimension of $5.5 \mathrm{~mm} \times 60 \mathrm{~mm}$. By controlling the phasing of klystron, $N_{\|}$can be controlled from 1.9 to 3.1 .

We used two techniques to evaluate RF coupling of LH3 launcher. The one is to use versatile FEM-CAE package (COMSOL) to solve an entire plasma-antenna coupling problem [10], and the other is to split the coupling problem to the vacuum region problem and the plasma region problem, and to combine two independently obtained solutions by cascading RF S-matrices [3]. In the latter approach, we used COMSOL for the vacuum region problem and the ALOHA code [21] for the plasma region problem. The second approach is computationally efficient and is used in the most of parametric survey performed during design process. However, since the angle between the

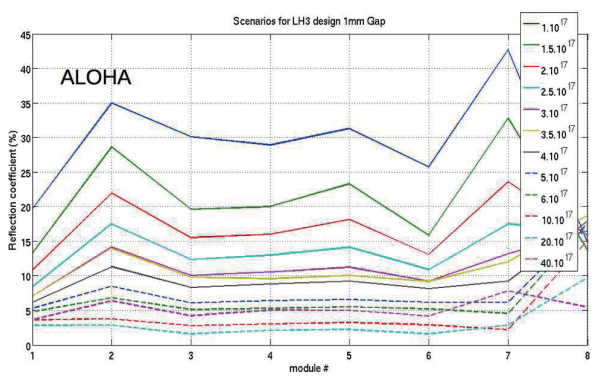

Fig. 6 Power reflection coefficient evaluated at feeding ports of 8 -way splitters. The vacuum problem is solved by using COMSOL and the plasma-antenna coupling is solved by ALOHA. The linear density gradient with $1 \mathrm{~mm}$ of vacuum gap [22] was used and the simulations were performed for various edge density. The cut-off density for $4.6 \mathrm{GHz}$ corresponds $2.6 \times 10^{17} \mathrm{~m}^{-3}$.

horizontal waveguide of 8-way splitter and the magnetic flux surface is rather shallow, the wave propagation may not be well captured by a stratified plasma approximation used in ALOHA. To address this issue, we modelled a small section of plasma facing waveguide precisely using the first approach and narrowed the waveguide opening to avoid the excitation of a higher order mode at the opening. Then, the first order effect of curved facing surface is introduced as an additional phase shift in the S-matrix of the antenna-plasma coupling, which is then used in the second approach. Figure 6 shows the reflection coefficient at the feeding port of 8-way splitter for various plasma density in front of launcher. A good coupling performance is predicted for all 8 feeding ports, showing that the power reflection is below $10 \%$ when the density in front of the launcher is higher than the cut-off density $\left(3 \times 10^{17} \mathrm{~m}^{-3}\right)$. Compared to LH2, thanks to bi-junctions and other optimization discussed in detail in Ref. [23], the predicted power reflection is smaller by a factor of 2-3.

\section{Summary}

Alcator C-Mod is planning to increase its LHCD power to $2 \mathrm{MW}$ from present $1 \mathrm{MW}$ level, by adding a new LH launcher (LH3). Parasitic edge power losses are considered to cause unexpectedly large degradation of LH efficiency at high density. LH3 aims to minimize such losses by enhancing strong single pass power absorption, and its result would have a crucial importance both for future AT experiments on C-Mod and LHCD on ITER. In order to realize strong single pass power absorption, LH3 is designed to maximize synergistic effects between the existing $\mathrm{LH} 2$ launcher. By placing the launcher off-midplane and selecting the launched $N_{\|}$higher than LH2, significant reduction of parasitic edge power absorption is predicted. RF coupler for LH3 combines the bi-junction developed for multi-junction type launcher [24] with the four-way splitter concept used in $\mathrm{LH} 2$, and good coupling performance (over $90 \%$ of power transmission) is predicted. 


\section{Acknowledgement}

This work was supported by US Department of Energy collaborative agreements DE-FC02-99ER54512 and DE-AC02-09CH11466 and SBIR grant award DE-FG0207ER84762.

[1] M. Porkolab, B. Lloyd, Y. Takase et al., Phys. Rev. Lett. 53, 1229 (1984).

[2] G. Hoang, A. Becoulet, J. Jacquinot et al., Nucl. Fusion 49, 075001 (2009).

[3] S. Shiraiwa, O. Meneghini, R. Paker et al., Nucl. Fusion 51, 103024 (2011).

[4] G.M. Wallace, A.E. Hubbard, P.T. Bonoli et al., Nucl. Fusion 51, 083032 (2011)

[5] G. Wallace, I.C. Faust, O. Meneghini et al., Phys. Plasmas 19, 062505 (2012).

[6] A. Tuccillo, Y. Baranov, R. Cesario et al., submitted to Nucl. Fusion.

[7] A.P. Smirnov and R. Harvey, Bull. Am. Phys. Soc. 40, 1837 (1995).

[8] R.W. Harvey and M. McCoy, in Proc. of the IAEA TCM on Simulation and Modeling of Thermonuclear Plasmas, Montreal, pp.489-526 (1992).

[9] O. Meneghini, S. Shiraiwa and R. Parker, Phys. Plasmas 16, 090701 (2010).

[10] S. Shiraiwa, O. Meneghini, R. Parker et al., Phys. Plasmas
17, 056119 (2010).

[11] S.G. Baek et al., accepted for publication in Plasma Phys. Control. Fusion.

[12] O. Meneghini, Ph. D. thesis (2011).

[13] Y. Takase, M. Porkolab, J.J. Schuss et al., Phys. Fluids 28, 983 (1985).

[14] P.T. Bonoli, R.R. Parker, M. Porkolab et al., Nucl. Fusion 40, 1251 (2000).

[15] D.G. Whyte, A.E. Hubbard, J.W. Hughes et al., Nucl Fusion 50, 105005 (2010).

[16] R. Cesario, A. Cardinali, C. Castaldo et al., Phys. Rev. Lett. 92, 175002 (2004).

[17] R.J. Dumont and G. Giruzzi, Phys. Plasmas 11, 3449 (2004).

[18] K. Nakamura, S. Itoh, H. Zushi et al., Nucl. Fusion 42, 340 (2002).

[19] S. Ide, O. Naito, T. Kondoh et al., Phys. Rev. Lett. 73, 2312 (1994).

[20] P.T. Bonoli and E. Ott, Phys. Fluids 25, 359 (1982).

[21] J. Hillairet, D. Voyer, A. Ekedahl et al., Nucl. Fusion 50, 125010 (2010).

[22] J. Stevens J., M. Ono, R. Horton and J.R. Wilson, Nucl. Fusion 21, 125964 (1981).

[23] G.M. Wallace et al., submitted to Nucl. Fusion.

[24] C. Gormezano, P. Briand, G. Briffod et al., Nucl. Fusion 25, 419 (1985). 